\title{
TTTAN'S ATMOSPHERE PROBED BY STELLAR OCCULTATION
}

\author{
A. Brahic ${ }^{\star}+$ B. Sicardy ${ }^{\star}$, C. Ferrarit, and D. Gautiert \\ tObservatoire de Paris, France \\ *Universite Paris VII, France \\ Paper Presented by A. Brahic
}

Summary

Saturn's giant moon Titan shares with the Earth and Triton a predominantly nitrogen atmosphere. Most of current information about Titan's atmosphere was obtained by Voyager I fly-by on November 12, 1980. This satellite will remain a mysterious object until the ESA-NASA Cassini-Huygens mission. Titan's atmosphere may be conveniently divided into three regions: (i) the troposphere (with pressure $>\sim 50 \mathrm{mbar}$, or an altitude $<\sim 50 \mathrm{~km}$ ), dominated by convection, (ii) the middle atmosphere, or stratosphere (from $~-50$ mbar to $\sim 5$ nbar, i.e. between -50 and $800 \mathrm{~km}$ ), dominated by radiative equilibrium, and (iii) the thermosphere and the exosphere (above the -5 nbar level), dominated by conduction. The Voyager I radio occultation experiment probed the atmosphere of Titan's ground until an altitude of $200 \mathrm{~km}$, as did the infrared spectrometer. On the other hand, the Voyager UV spectrometer explored higher levels in the thermosphere, between 1000 and $1300 \mathrm{~km}$ altitude. Many physical processes (heating, cooling, haze variations, vibrational relaxation of molecules, ionization phenomena, molecular absorption, organic condensation,...) are involved in the region between $1 \mathrm{mbar}$ and $1 \mathrm{nbar}$, where the temperature profile is uncertain. This is why a direct measurement is important.

On July 3, 1989, the bright star 28 Sagitarii, or SAO $187255(\mathrm{~V}-5.5)$, passed behind the satellite $(V \sim 8.3)$. The event was visible from Europe, North America and Middle-East. This observation probed Titan's atmosphere in the $\sim 250-500 \mathrm{~km}$ altitude range, or the $-250-1 \mu$ bar pressure range, where up to now there was an "information gap" between infrared and UV Voyager observations. This was the first stellar occultation by Titan ever observed. B. Sicardy, C. Ferrari, J. Lecacheux, F. Roques, J. Arlot, F. Colas, W. Thuillot, F. Sevres, J.L. Vidal, C. Blanco, S. Cristaldi, C. Buil, A. Kletz and E. Thouvenot observed this occultation at Paris Observatory and Catania Observatory.

These observations provide information on Titan's stratosphere between the 250 and $500 \mathrm{~km}$ altitude levels. We derive a mean-scale-height of $48+/-3 \mathrm{~km}$ at $450 \mathrm{~km}$ altitude ( $3 \mu$ bar pressure level). This constrains the mean temperature to be in the range $149<\mathrm{T}<178 \mathrm{~K}$ at that level. Titan's shadow center passed within a few tens of kilometers of Paris, and the central flash observed there is an unique opportunity to constrain the apparent oblateness of Titan's atmosphere at the 0.25 mbar level (250 km altitude), giving a value which may be as high as 0.014 . This atmospheric oblateness could be due to a super rotation of the atmosphere or a differential thermal effect. If entirely due to rotation, the oblateness would require a minimum 26 -hour atmospheric rotation period which is much smaller than the solid body rotation period (assumed to rotate with the synchronous period of about 16 days). Then Titan's large scale stratospheric motions may be of the same class as those of Venus. 